\title{
Fully Adaptive Space-Time Processing on Nested Arrays
}

\author{
Peter Vouras \\ Naval Research Laboratory, Radar Division \\ Washington, DC 20375
}

\begin{abstract}
This paper describes fully adaptive nonlinear space-time processing on nested arrays. Nested arrays are sparse arrays that in conjunction with nonlinear processing of the received signals enable more adaptive degrees of freedom than a conventional linear beamformer would allow. The Space-Time Adaptive Processing (STAP) architecture proposed in this paper consists of a nested array that samples received pulses on a nested temporal interval. The sparse spatial and temporal sampling pattern of the proposed architecture together with the nonlinear processing performed on the received pulses makes this STAP architecture fundamentally different from conventional STAP arrays. Simulated results for an airborne platform illustrate angle-Doppler nulling performance for illuminated ground clutter and for jamming interference.
\end{abstract}

\section{OVERVIEW OF STAP ON NESTED ARRAYS}

An existing body of research describes STAP on sparse antenna arrays with applications to space-borne radars that are often designed to satisfy stringent size, weight, and power constraints by minimizing the total number of array elements [1]. Quite often these antenna designs will exhibit spatial grating lobes along with a limited number of adaptive degrees of freedom precisely because of their sparsity. Recently, nonlinear adaptive beamforming techniques performed on a specific type of sparse arrays, called nested arrays, have been described which offer enhanced adaptive degrees of freedom and eliminate grating lobes. Nested linear arrays are nonuniform passive arrays created by concatenating two or more uniform linear arrays (ULAs) to create an array with increasing inter-sensor spacing. By nonlinearly pre-processing the received signals and directly adapting their second order statistics across the array aperture, it is possible for nested arrays to obtain $O\left(N^{2}\right)$ adaptive degrees of freedom using $N$ physical sensors [2]-[6]. For instance, a two-level nested linear array of length 12 is an array with 6 elements located at the positions $\{0,1,2,3,7,11\}$. The smallest spacing between the elements of a nested array corresponds to one-half the wavelength of the highest received frequency to avoid spatial aliasing.

Consider an $N$ element nested linear array and let $r_{i}$ for $i=0, \ldots, N-1$ denote the position of the $i$ th sensor, which is an integer multiple of $\lambda / 2$. The $N$-by-1 steering vector $\mathbf{v}(\phi)$ corresponding to the direction $\phi$ is,

$$
\mathbf{v}(\phi)=\left[\begin{array}{ll}
1 & e^{j(2 \pi / \lambda) r_{1} \sin \phi} \ldots e^{j(2 \pi / \lambda) r_{N-1} \sin \phi}
\end{array}\right]^{T} .
$$

In addition, given a fixed radar range bin, let the sequence $\left\{d_{j}\right\}$ for $j=0, \ldots, M-1$ denote the $M$ pulses for a nested temporal interval. The Doppler steering vector $\mathbf{b}(\theta)$ corresponding to a radial velocity $v$ of a signal source can be written as

$$
\mathbf{b}(\theta)=\left[\begin{array}{ll}
1 & e^{j 2 \pi \theta d_{1}} \ldots e^{j 2 \pi \theta d_{N-1}}
\end{array}\right]^{T} .
$$

Here the normalized Doppler frequency $\theta$ is given by

$$
\theta=\frac{2 v}{\lambda} T_{p}=\frac{f_{d}}{f_{r}}
$$

where $T_{p}$ is the smallest pulse repetition interval (PRI) between pulses, $f_{d}$ is the Doppler frequency shift, and $f_{r}$ is the pulse repetition frequency (PRF). To avoid temporal aliasing, $T_{p}$ should be chosen so that $-0.5 \leq \theta \leq 0.5$. The space-time steering vector for the azimuth direction $\phi$ and the normalized Doppler frequency $\theta$ is defined as

$$
\mathbf{a}(\phi, \theta)=\mathbf{b}(\theta) \otimes \mathbf{v}(\phi)
$$

where $\otimes$ denotes the Kronecker product. In the sequel, the notation $\mathbf{a}_{c}(\phi, \beta \phi)$ denotes the Kronecker product

$$
\mathbf{a}_{c}(\phi, \beta \phi)=\mathbf{v}(\phi)^{\beta} \otimes \mathbf{v}(\phi)
$$

where $\mathbf{v}(\phi)^{\beta}=\left[\begin{array}{ll}1 & e^{j \beta(2 \pi / \lambda) r_{1} \sin \phi} \ldots e^{j \beta(2 \pi / \lambda) r_{N-1} \sin \phi}\end{array}\right]^{T}$.

Assuming that clutter, jamming, and thermal noise sources are all mutually uncorrelated, the interference plus noise space-time covariance matrix is given by

$$
\mathbf{R}_{i+n}=\mathbf{R}_{c}+\mathbf{R}_{j}+\mathbf{R}_{n}
$$

where $\mathbf{R}_{n}$ is the noise covariance matrix, $\mathbf{R}_{j}$ is the jamming covariance matrix, and $\mathbf{R}_{c}$ is the clutter covariance matrix. If the thermal noise processes at each array element are mutually uncorrelated and noise samples at a single array element separated by multiples of $T_{p}$ are temporally uncorrelated, then

$$
\mathbf{R}_{n}=\sigma_{n}{ }^{2} \mathbf{I}_{M N}
$$

where $\sigma_{n}^{2}$ is the noise power (normalized to unity) and $\mathbf{I}_{M N}$ is the $M \times N$ identity matrix.

For the case of barrage noise jamming originating at long range from a land-based or airborne platform, it is assumed that the jamming is spatially correlated from array element to array element but temporally uncorrelated from pulse to pulse. Thus, the jamming looks like thermal noise temporally, but like a point target in the spatial domain. For a single jammer 
in the direction $\phi_{1}$, the jamming covariance matrix becomes

$$
\mathbf{R}_{j}=\mathbf{A}_{j}\left[\begin{array}{cccc}
\sigma_{j_{1}}{ }^{2} & 0 & \cdots & 0 \\
0 & \sigma_{j_{2}}{ }^{2} & \ddots & \vdots \\
\vdots & \ddots & \ddots & 0 \\
0 & \ldots & 0 & \sigma_{j_{D}}{ }^{2}
\end{array}\right] \mathbf{A}_{j}^{H}
$$

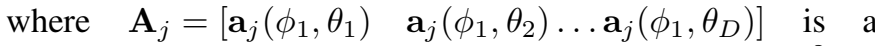
matrix of $D$ jammer space-time steering vectors and $\sigma_{j_{k}}^{2}$ is the power of the jammer at the $k$ th Doppler frequency [7]. For the assumed case of temporally uncorrelated jamming, $\sigma_{j_{k}}^{2}=\sigma_{j}^{2}$ for all $k$.

Since the STAP processor is deployed on an airborne radar that illuminates patches of ground clutter, the motion of the aircraft imparts a Doppler shift to each clutter patch that depends on azimuth angle. The total clutter return for a fixed range can be modeled as the superposition of the contributions from many independent clutter sources evenly distributed in azimuth about the radar. The result is that the received clutter forms a diagonal ridge in the Doppler-azimuth output of the STAP processor which may mask legitimate targets. Assuming the radar illuminates $K$ independent clutter sources, the clutter covariance matrix may be written as [8]

$$
\mathbf{R}_{c}=\mathbf{A}_{c}\left[\begin{array}{cccc}
\sigma_{c_{1}}{ }^{2} & 0 & \cdots & 0 \\
0 & \sigma_{c_{2}}{ }^{2} & \ddots & \vdots \\
\vdots & \ddots & \ddots & 0 \\
0 & \ldots & 0 & \sigma_{c_{K}}^{2}
\end{array}\right] \mathbf{A}_{c}^{H}
$$

where in this case $\sigma_{c_{k}}^{2}$ is the power of the $k$ th clutter source and $\mathbf{A}_{c}=\left[\mathbf{a}_{c}\left(\phi_{1}, \beta \phi_{1}\right) \quad \mathbf{a}_{c}\left(\phi_{2}, \beta \phi_{2}\right) \ldots \mathbf{a}_{c}\left(\phi_{K}, \beta \phi_{K}\right)\right]$. Note that the clutter Doppler shift is proportional to the clutter spatial frequency for each space-time steering vector. The parameter $\beta$ represents the constant of proportionality.

If a target is present in the received data, then the total signal covariance matrix becomes

$$
\mathbf{R}=\sigma_{t}^{2} \mathbf{a}_{t}\left(\phi_{t}, \theta_{t}\right) \mathbf{a}_{t}\left(\phi_{t}, \theta_{t}\right)^{H}+\mathbf{R}_{i+n}
$$

where $\sigma_{t}^{2}$ is the power of the target in direction $\phi_{t}$ with velocity corresponding to $\theta_{t}$. To form the adapted output of the STAP processor, the covariance matrix $\mathbf{R}$ is vectorized to form the $(M N)^{2} \times 1$ vector $\mathbf{z}$ (here the subscripts $c, j$, and $t$ corresponding to clutter, jamming, and target will be dropped),

$$
\begin{aligned}
\mathbf{z} & =\operatorname{vec}(\mathbf{R}) \\
& =\operatorname{vec}\left[\sum_{i=1}^{D+K+1} \sigma_{i}{ }^{2}\left(\mathbf{a}\left(\phi_{i}, \theta_{i}\right) \mathbf{a}\left(\phi_{i}, \theta_{i}\right)^{H}\right)\right]+\sigma_{n}{ }^{2} \mathbf{1}_{n} \\
& =\left(\mathbf{A}^{*} \odot \mathbf{A}\right) \mathbf{p}+\sigma_{n}{ }^{2} \mathbf{1}_{n}
\end{aligned}
$$

where $^{*}$ denotes conjugation, $\mathbf{p}=\left[\begin{array}{lll}\sigma_{1}{ }^{2} & {\sigma_{2}}^{2} \ldots \sigma_{D+K+1}{ }^{2}\end{array}\right]^{T}$, $\mathbf{1}_{n}=\left[\begin{array}{ll}\mathbf{e}_{1}^{T} & \mathbf{e}_{2}{ }^{T} \ldots \mathbf{e}_{M N}{ }^{T}\end{array}\right]^{T}$ and $\mathbf{e}_{i}$ is a column vector of all zeros except for a one in the $i$ th position. The matrix

$$
\begin{aligned}
& \mathbf{A}^{*} \odot \mathbf{A}= \\
& {\left[\mathbf{a}\left(\phi_{1}, \theta_{1}\right)^{*} \otimes \mathbf{a}\left(\phi_{1}, \theta_{1}\right) \cdots\right.} \\
& \left.\cdots \mathbf{a}\left(\phi_{D+K+1}, \theta_{D+K+1}\right)^{*} \otimes \mathbf{a}\left(\phi_{D+K+1}, \theta_{D+K+1}\right)\right]
\end{aligned}
$$

is the Khatri-Rao product of the matrices $\mathbf{A}^{*}$ and $\mathbf{A}$. The nonlinearly adapted output of the STAP processor is formed by determining a weight vector $\mathbf{w}$ and computing the inner product $\mathbf{w}^{H} \mathbf{z}$.

\section{AdAPtive Weight COMPUtation; BEAMFORMER 1}

This section describes an approach for computing an adaptive weight vector which is analogous to the Minimum Variance Distortionless Response (MVDR) beamformer commonly applied to conventional arrays. For the beamformers derived in this paper it is assumed that the clutter does not have any Doppler spread due to internal motion. The first beamformer proceeds by minimizing the contribution to the array output power from interference and clutter sources. The optimal weight vector $\mathbf{w}$ is the solution to the optimization program

$$
\begin{aligned}
& \operatorname{minimize} \mathbf{w}^{H} \mathbf{Q w} \\
& \text { such that } \quad \mathbf{w}^{H}\left[\mathbf{a}\left(\phi_{0}, \theta_{0}\right)^{*} \otimes \mathbf{a}\left(\phi_{0}, \theta_{0}\right)\right]=1 .
\end{aligned}
$$

The kernel matrix

$$
\begin{aligned}
& \mathbf{Q}= \\
& \sum_{k=1}^{K} \sigma_{c_{k}}^{2}\left[\mathbf{a}_{c}\left(\phi_{k}, \beta \phi_{k}\right)^{*} \otimes \mathbf{a}_{c}\left(\phi_{k}, \beta \phi_{k}\right)\right]\left[\mathbf{a}_{c}\left(\phi_{k}, \beta \phi_{k}\right)^{*} \otimes \mathbf{a}_{c}\left(\phi_{k}, \beta \phi_{k}\right)\right]^{H} \\
& +\sum_{p=1}^{D} \sigma_{j}^{2}\left[\mathbf{a}_{j}\left(\phi_{1}, \theta_{p}\right)^{*} \otimes \mathbf{a}_{j}\left(\phi_{1}, \theta_{p}\right)\right]\left[\mathbf{a}_{j}\left(\phi_{1}, \theta_{p}\right)^{*} \otimes \mathbf{a}_{j}\left(\phi_{1}, \theta_{p}\right)\right]^{H}
\end{aligned}
$$

is formed over all jamming frequencies and clutter sources. The single constraint in (13) corresponds to the desired mainbeam pointing direction $\left(\phi_{0}, \theta_{0}\right)$ in angle-Doppler space. The matrix $\mathbf{Q}$ will not be full rank because of the redundant phase terms in each Kronecker product. To find the optimum $\mathbf{w}$, first compute the singular value decomposition of $\mathbf{Q}$ as in

$$
\mathbf{Q}=\left[\begin{array}{ll}
\mathbf{U}_{1} & \mathbf{U}_{2}
\end{array}\right]\left[\begin{array}{cc}
\boldsymbol{\Sigma} & \mathbf{0} \\
\mathbf{0} & \mathbf{0}
\end{array}\right]\left[\begin{array}{c}
\mathbf{V}_{1}^{H} \\
\mathbf{V}_{2}^{H}
\end{array}\right]
$$

If the rank of $\mathbf{Q}$ is $r$, then the associated matrix sizes are; $\mathbf{U}_{1}$ is $(M N)^{2} \times r, \mathbf{U}_{2}$ is $(M N)^{2} \times\left((M N)^{2}-r\right), \mathbf{V}_{1}$ is $(M N)^{2} \times r, \mathbf{V}_{2}$ is $(M N)^{2} \times\left((M N)^{2}-r\right)$, and $\boldsymbol{\Sigma}$ is $r \times r$. Note that since $\mathbf{Q}$ is Hermitian, $\mathbf{U}_{1}=\mathbf{V}_{1}$. Following the approach described in [9], the optimal weight vector may be computed as

$$
\begin{aligned}
& \mathbf{w}_{O P T}= \\
& \frac{\mathbf{Q}^{\dagger}\left[\mathbf{a}\left(\phi_{0}, \theta_{0}\right)^{*} \otimes \mathbf{a}\left(\phi_{0}, \theta_{0}\right)\right]}{\left[\mathbf{a}\left(\phi_{0}, \theta_{0}\right)^{*} \otimes \mathbf{a}\left(\phi_{0}, \theta_{0}\right)\right]^{H} \mathbf{Q}^{\dagger}\left[\mathbf{a}\left(\phi_{0}, \theta_{0}\right)^{*} \otimes \mathbf{a}\left(\phi_{0}, \theta_{0}\right)\right]}
\end{aligned}
$$

where the pseudoinverse of $\mathbf{Q}$ is equal to the matrix $\mathbf{Q}^{\dagger}=\mathbf{V}_{1} \boldsymbol{\Sigma}^{-1} \mathbf{V}_{1}^{H}$. A drawback to this beamformer solution is 
that it may not force the ambient sidelobe levels to adequately low levels.

An approach for improving the sidelobe response of the adapted array is to load the diagonal of the kernel matrix $\mathbf{Q}$ as in

$$
\begin{aligned}
& \operatorname{minimize} \mathbf{w}^{H}\left[\mathbf{Q}+\gamma^{2} \mathbf{I}\right] \mathbf{w} \\
& \text { such that } \mathbf{w}^{H}\left[\mathbf{a}\left(\phi_{0}, \theta_{0}\right)^{*} \otimes \mathbf{a}\left(\phi_{0}, \theta_{0}\right)\right]=1 .
\end{aligned}
$$

where $\gamma^{2}$ is a positive, real scalar. In this case, the matrix $\mathbf{H}=\mathbf{Q}+\gamma^{2} \mathbf{I}$ is full rank and the standard analytical solution for a quadratic optimization function with linear constraints applies, [10]

$$
\begin{aligned}
& \mathbf{w}_{O P T}= \\
& \frac{\mathbf{H}^{-1}\left[\mathbf{a}\left(\phi_{0}, \theta_{0}\right)^{*} \otimes \mathbf{a}\left(\phi_{0}, \theta_{0}\right)\right]}{\left[\mathbf{a}\left(\phi_{0}, \theta_{0}\right)^{*} \otimes \mathbf{a}\left(\phi_{0}, \theta_{0}\right)\right]^{H} \mathbf{H}^{-1}\left[\mathbf{a}\left(\phi_{0}, \theta_{0}\right)^{*} \otimes \mathbf{a}\left(\phi_{0}, \theta_{0}\right)\right]} .
\end{aligned}
$$

\section{AdAPtive Weight COMPUtATion; BeAmFormer 2}

If the directions $\phi_{k}$ and Dopplers $\theta_{k}$ of all jamming and clutter sources are known or can be estimated, then a superior beamforming solution can be obtained which places very deep nulls in desired locations and reduces the ambient sidelobe level of the adapted pattern. The optimization program to be solved is

$$
\begin{aligned}
& \operatorname{minimize} \mathbf{w}^{H} \mathbf{Q w} \\
& \text { such that } \mathbf{G}^{H} \mathbf{w}=\mathbf{b} .
\end{aligned}
$$

In this formulation the matrix $\mathbf{Q}$ becomes,

$\mathbf{Q}=$

$\sum_{k=1}^{N_{1}} \sum_{m=1}^{N_{2}}\left[\mathbf{a}\left(\phi_{k}, \theta_{m}\right)^{*} \otimes \mathbf{a}\left(\phi_{k}, \theta_{m}\right)\right]\left[\mathbf{a}\left(\phi_{k}, \theta_{m}\right)^{*} \otimes \mathbf{a}\left(\phi_{k}, \theta_{m}\right)\right]^{H}$,

and the summation is over all possible azimuths and Doppler frequencies sampled on a discrete grid. The constraint matrix corresponding to nulls in the directions of jamming and clutter sources and unity gain in the desired mainbeam pointing direction is given by

$$
\begin{aligned}
& \mathbf{G}^{H} \mathbf{w}= \\
& {\left[\begin{array}{c}
\left(\mathbf{a}\left(\phi_{0}, \theta_{0}\right)^{*} \otimes \mathbf{a}\left(\phi_{0}, \theta_{0}\right)\right)^{H} \\
\left(\mathbf{a}\left(\phi_{1}, \theta_{1}\right)^{*} \otimes \mathbf{a}\left(\phi_{1}, \theta_{1}\right)\right)^{H} \\
\vdots \\
\left(\mathbf{a}\left(\phi_{D+K}, \theta_{D+K}\right)^{*} \otimes \mathbf{a}\left(\phi_{D+K}, \theta_{D+K}\right)\right)^{H}
\end{array}\right] \mathbf{w}=\left[\begin{array}{c}
1 \\
0 \\
\vdots \\
0
\end{array}\right] .}
\end{aligned}
$$

The optimum solution is given by [9]

$$
\mathbf{w}_{O P T}=\mathbf{Q}^{\dagger} \mathbf{G}\left[\mathbf{G}^{H} \mathbf{Q}^{\dagger} \mathbf{G}\right]^{-1} \mathbf{b} .
$$

Reference [9] also describes an iterative algorithm which converges to this solution.

\section{RESUlts}

\section{A. Adapted Array Responses}

This section describes simulated results for both beamformers when applied to a nested STAP architecture with 6 array elements located at positions $\{0,1,2,3,7,11\}$ and 6 pulses sampled at the PRIs $\{0,1,2,3,7,11\}$. The operating frequency of the radar is $3.125 \mathrm{GHz}$ and the $\mathrm{PRF}$ is $2 \mathrm{kHz}$ which results in a maximum unambiguous radial velocity of $48 \mathrm{~m} / \mathrm{sec}$. There is one ground based jammer located at $24.9^{\circ}$. Twenty uniformly spaced Doppler frequencies were used to form the jammer covariance matrix described in (8) with $\sigma_{j_{k}}^{2}=1000$ for all $k$. Also, 45 clutter patches uniformly distributed in azimuth were used to form the clutter covariance matrix in (9) with $\sigma_{c_{k}}^{2}=10$ for all $k$. The slope $\beta$ of the clutter ridge was set equal to unity. In the results that follow the adapted output of the STAP processor is defined as $10 \log _{10}\left|\mathbf{w}^{H} \mathbf{z}\right|$.

Figure 1 illustrates the quiescent pattern formed by applying a uniform weighting to the array space-time steering vectors over all azimuths and Doppler frequencies. The quiescent array pattern shows a high ambient sidelobe level which may also be present in the adapted pattern depending on the beamformer solution chosen. Figure 2 illustrates the adapted array response constructed using Beamformer 1 with the mainbeam steered to $50.1^{\circ}$ in azimuth and a normalized Doppler frequency of 0.155 . The beamformer places visible nulls along the clutter ridge and the jammer output as desired, but the pattern lacks a clear peak in the desired mainbeam pointing direction. However, by applying diagonal loading with the parameter $\gamma^{2}=0.1$ the mainbeam response is more clearly visible as in Fig. 3.

Figure 4 illustrates the adapted array response constructed using Beamformer 2. Similar to Beamformer 1, 45 clutter patches and 20 Doppler frequencies were used to form the clutter and jamming covariance matrices respectively. The adapted array pattern clearly shows nulls along the clutter ridge and jamming interference along with a distinct mainbeam response.

\section{B. Simulated Receive Processing}

This section describes the adapted STAP output using simulated receive data from a multichannel nested array. An adaptive weight vector is calculated using Beamformer 2 with the assumption that the jamming and clutter sources are known clairvoyantly. Behind each simulated array element is an independent receiver that downconverts and samples the impinging signal. The simulation proceeds by generating the received signal sample at time instant $n$,

$$
x_{i k}[n]=x_{t_{i k}}[n]+x_{c_{i k}}[n]+x_{j_{i k}}[n]+x_{n_{i k}}[n]
$$

where $t, c, j$, and $n$ refer to target, clutter, jamming, and thermal noise respectively. The subscripts $i$ and $k$ belong to the nested set $\{0,1,2,3,7,11\}$ and refer to the $i$ th PRI of the $k$ th array element. Let $y[n, \eta]$ denote the complex envelope of a linear FM pulse sampled at time instant $n$ with initial phase 
$\eta$

$$
y[n, \eta]=\alpha \exp \left[j\left(\mu\left(n T_{s}\right)^{2} / 2+\eta\right)\right]
$$

where $T_{s}$ refers to the sampling interval and $\mu$ is the frequency sweep rate (in radians/sec). The scalar $\alpha$ is chosen such that $y[n]$ has unit energy. The $i k$ th target pulse $x_{t_{i k}}[n]$ is constructed as

$$
x_{t_{i k}}[n]=\sigma_{t_{i k}} y\left[n, \eta_{t_{i k}}\right]
$$

where ${\sigma_{t_{i k}}}^{2}$ equals the desired signal-to-noise ratio (SNR) of the pulse and $\eta_{t_{i k}}$ is the desired initial phase. The return from a single clutter patch is constructed in a similar manner. The summation over a total of $K$ clutter patches yields a single clutter pulse,

$$
x_{c_{i k}}[n]=\sum_{m=1}^{K} \sigma_{c_{i k m}} y\left[n, \eta_{c_{i k m}}\right]
$$

where $\sigma_{c_{i k m}}^{2}$ equals the desired clutter-to-noise ratio (CNR) for the $m$ th clutter patch and $\eta_{c_{i k m}}$ equals the desired initial phase.

The jamming samples are generated as

$$
x_{j_{i k}}[n]=\sigma_{j} \exp \left(j \eta_{j_{i k}}\right) n_{j}[n]
$$

where $n_{j}[n]$ is a complex sample of white noise with unity variance, $\sigma_{j}$ is the square root of the desired jamming-to-noise-ratio (JNR) and $\eta_{j_{i k}}$ corresponds to a phase shift. The complex thermal noise samples $x_{n_{i k}}[n]$ are independently generated for each $i$ and $k$ and have unity variance.

The phase terms $\eta_{t_{i k}}, \eta_{c_{i k m}}$, and $\eta_{j_{i k}}$ are set equal to the phase of the component in the target, clutter, or jamming space-time steering vector respectively which corresponds to the $i k$ th pulse. A target space-time steering vector as in (4) is created by traversing through all azimuths in $0.4^{\circ}$ increments and through all normalized Doppler frequencies in 0.0025 increments. The jamming space-time steering vector for a single jammer in the direction $\phi_{1}$ is given by

$$
\mathbf{a}_{j}\left(\phi_{1}\right)=\mathbf{1}_{M} \otimes \mathbf{v}\left(\phi_{1}\right)
$$

where the $M \times 1$ vector $\mathbf{1}_{M}=\left[\begin{array}{ll}1 & 1 \ldots 1\end{array}\right]^{T}$. The space-time steering vector for a single clutter patch was generated by

$$
\mathbf{a}_{c}\left(\phi_{k}, \beta \phi_{k}\right)=\mathbf{v}\left(\phi_{k}\right)^{\beta} \otimes \mathbf{v}\left(\phi_{k}\right)
$$

where the slope of the clutter ridge, $\beta=1$. The total number of clutter patches $K=76$ and the angles $\phi_{k}$ are uniformly distributed over all azimuths. The power $\sigma_{c_{i k m}}^{2}$ in (26) for each clutter patch was set equal to $10 \mathrm{~dB}$.

Once a PRI of uncompressed signal samples $x_{i k}[n]$ is created by summing together the target, jamming, clutter, and thermal noise contributions, the entire PRI is convolved with the reference pulse $y^{*}[n, 0]$ to produce the pulse compressed output, $r_{i k}[n]=x_{i k}[n] * y^{*}[n, 0]$. This output is then sampled at the target range bin $n_{0}$ to yield $r_{i k}\left[n_{0}\right]$. The pulse compressed target samples, $r_{i k}\left[n_{0}\right]$ (one per PRI), are then stacked in the sequence $\hat{\mathbf{a}}(\phi, \theta)=\left[r_{11}\left[n_{0}\right] \ldots r_{1 N}\left[n_{0}\right] \ldots r_{M 1}\left[n_{0}\right] \ldots r_{M N}\left[n_{0}\right]\right]$ to form an $M N \times 1$ space-time vector analogous to (4). A total of $L$ such vectors are used to form the vectorized sample space-time covariance matrix $\hat{\mathbf{z}}(\phi, \theta)=\operatorname{vec}\left[(1 / L) \sum \hat{\mathbf{a}}_{k}(\phi, \theta) \hat{\mathbf{a}}_{k}(\phi, \theta)^{H}\right]$ and finally the normalized adapted array output $10 \log _{10}\left|\mathbf{w}^{H} \hat{\mathbf{z}}(\phi, \theta)\right|$.

Figure 5 shows the output of Beamformer 1 for the case where the target SNR is equal to $20 \mathrm{~dB}$ and the main beam is steered to $50.1^{\circ}$ azimuth and a Doppler frequency of 0.155 . The JNR for a single jamming pulse was $14.87 \mathrm{~dB}$ and the average CNR for a single clutter pulse constructed by summing together the returns from 76 clutter patches was 27 dB. Figure 6 illustrates the matched filter output obtained by using the adaptive weight vector $\mathbf{w}(\phi, \theta)=\mathbf{a}(\phi, \theta)^{*} \otimes \mathbf{a}(\phi, \theta)$ and normalizing such that $\|\mathbf{w}\|^{2}=1$. Figures 7 and 8 illustrate azimuth and Doppler principal plane cuts at the target location. The red curve corresponds to the output of the target only signal without clutter, jamming, or noise present. The black curve corresponds to the matched filter output. Figures 9 and 10 show the STAP and matched filter outputs for Beamformer 2 with the target SNR equal to $60 \mathrm{~dB}$. Figures 11 and 12 depict the azimuth and Doppler principal planes. All results shown are for 80 data snapshots used to form the sample covariance matrix.

\section{CONCLUSION}

This paper describes fully adaptive space-time processing on nested arrays.

\section{ACKNOWLEDGEMENTS}

This work was carried out under the Naval Research Laboratory Base Program sponsored by the Office of Naval Research.

\section{REFERENCES}

[1] J. Ward, "Space-Time Adaptive Processing With Sparse Antenna Arrays," Proceedings of the Asilomar Conference on Signals, Systems, and Computers, Pacific Grove, CA., November 1-4, 1998.

[2] P. Pal and P. P. Vaidyanathan, "Nested Arrays: A Novel Approach to Array Processing with Enhanced Degrees of Freedom," IEEE Transactions on Signal Processing, Vol. 58, No. 8, August 2010.

[3] W. Ma, T. Hsieh, and C. Chi, "DOA Estimation of Quasi-Stationary Signals via Khatri-Rao Subspace," Proceedings of the International Conference on Acoustics, Speech, and Signal Processing (ICASSP), Taipei, Taiwan, April 19-24, 2009.

[4] R. T. Hoctor and S. Kassam, "The Unifying Role of the Coarray in Aperture Synthesis for Coherent and Incoherent Imaging," Proceedings of the IEEE, Volume 78, Issue 4, 1990.

[5] P. Pal and P. P. Vaidyanathan, "Nested Arrays in Two Dimensions, Part I: Geometrical Considerations," IEEE Transactions on Signal Processing, Vol. 60, No. 9, September 2012.

[6] P. Pal and P. P. Vaidyanathan, "Nested Arrays in Two Dimensions, Part II: Application in Two Dimensional Array Processing," IEEE Transactions on Signal Processing, Vol. 60, No. 9, September 2012.

[7] B. Friedlander, "A Subspace Method for Space Time Adaptive Processing," IEEE Transactions on Signal Processing, Vol. 53, No. 1, January 2005.

[8] J. Ward, "Space-Time Adaptive Processing for Airborne Radar," MIT Lincoln Laboratory Technical Report Nbr. 1015, December 1994.

[9] P. Vouras, "Sample Support Requirements for Nonlinear Adaptive Beamforming," Proceedings of the Conference on Information Sciences and Systems (CISS), Baltimore, MD., March 18-20, 2015.

[10] S. Haykin, Adaptive Filter Theory, 3rd ed. Upper Saddle River, NJ, USA: Prentice Hall, 1996. 


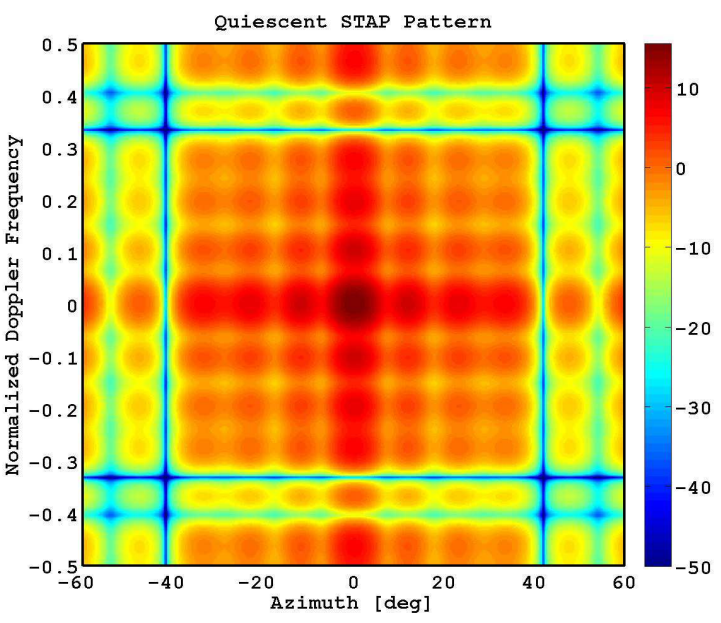

Fig. 1. Quiescent Pattern

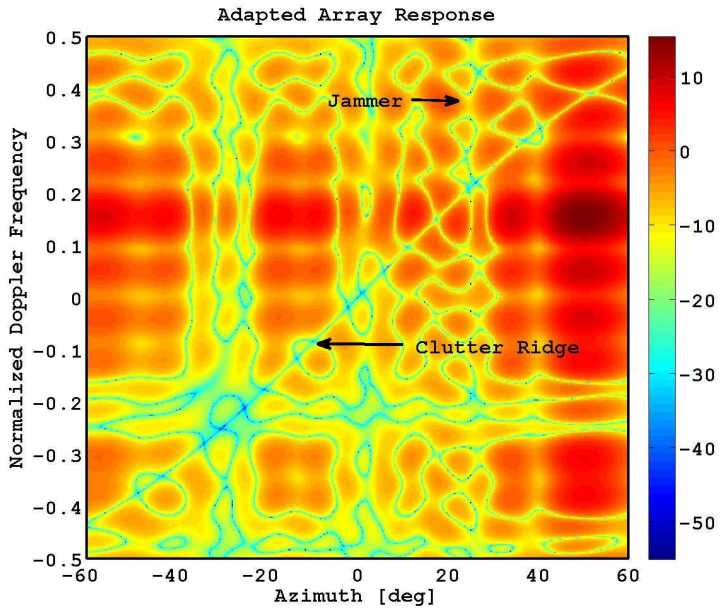

Fig. 3. STAP Pattern; Beamfomer $1, \gamma^{2}=0.1$

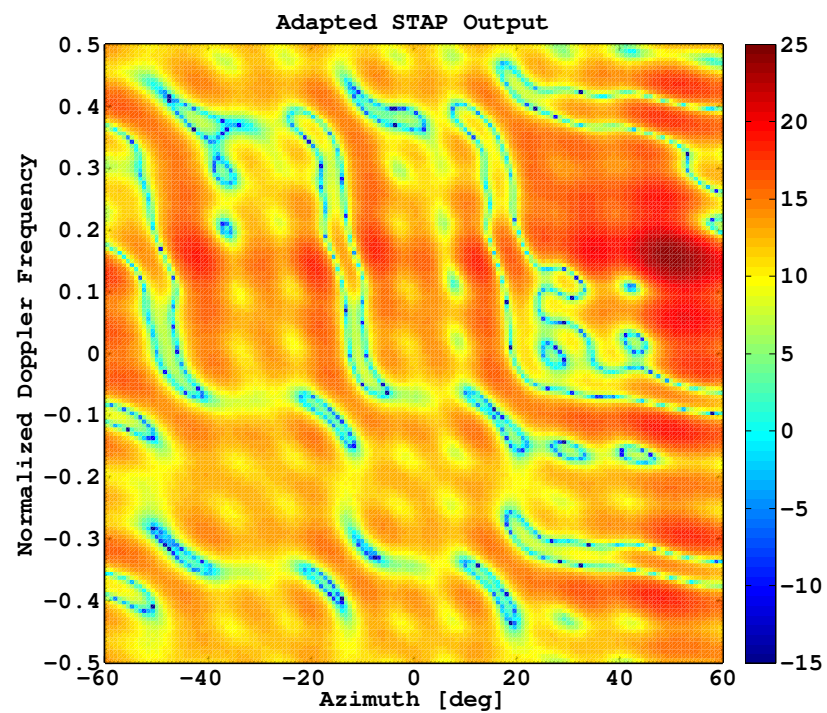

Fig. 5. STAP Output; Beamformer 1, SNR $=20 \mathrm{~dB}, 80$ Data Snapshots

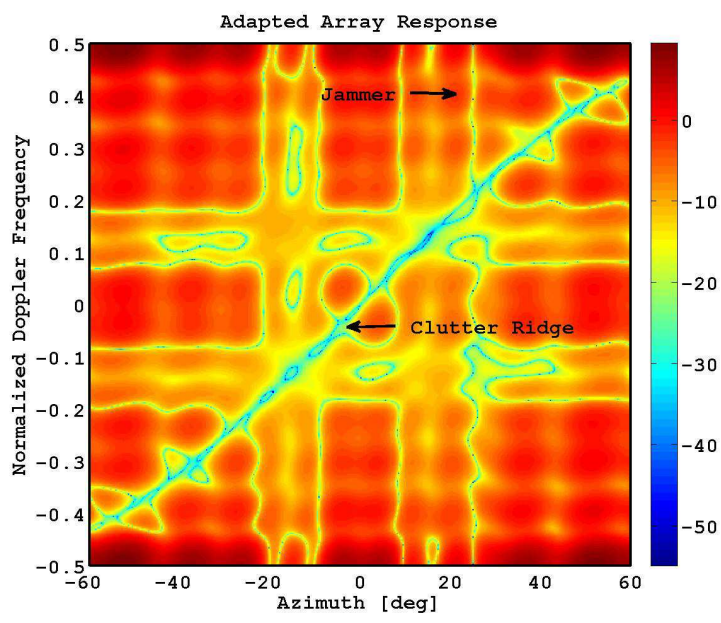

Fig. 2. STAP Pattern; Beamformer $1, \gamma^{2}=0$

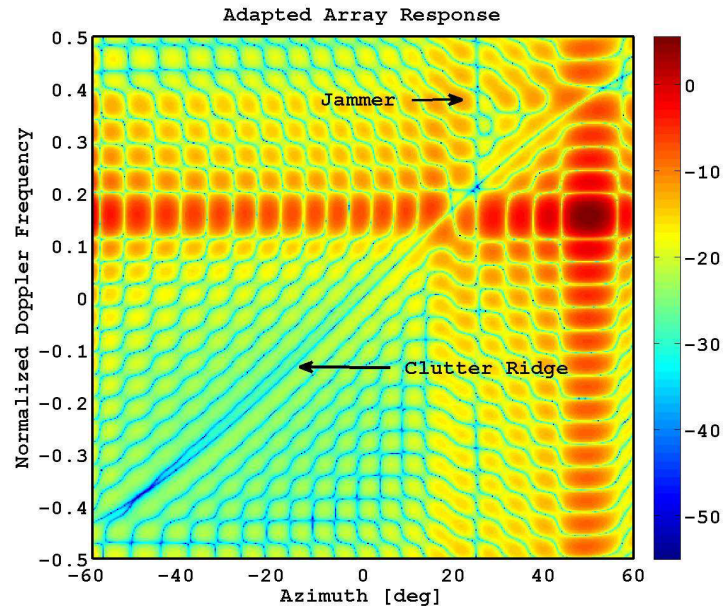

Fig. 4. STAP Pattern; Beamformer 2

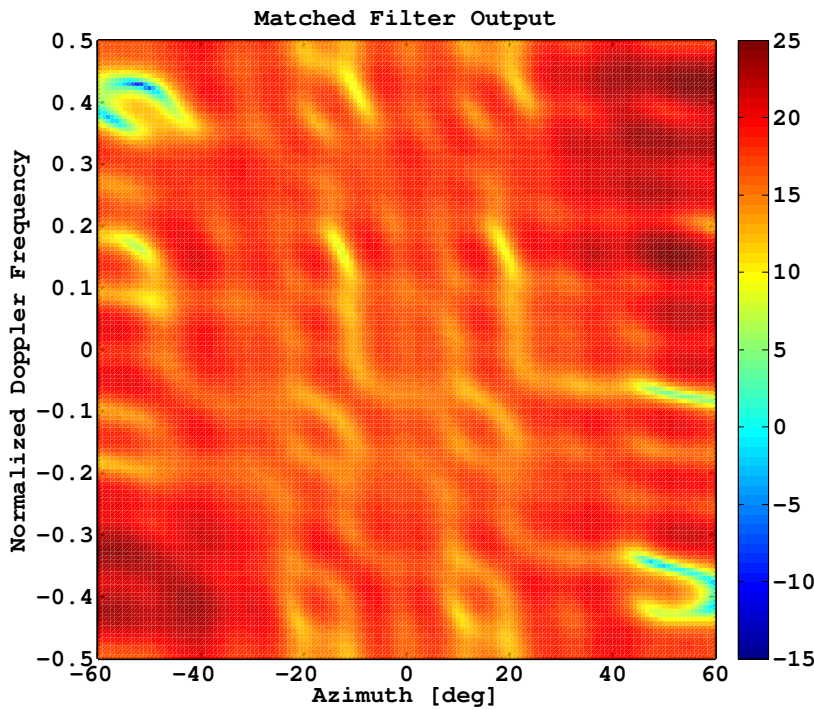

Fig. 6. Matched Filter Output; SNR $=20 \mathrm{~dB}, 80$ Data Snapshots 


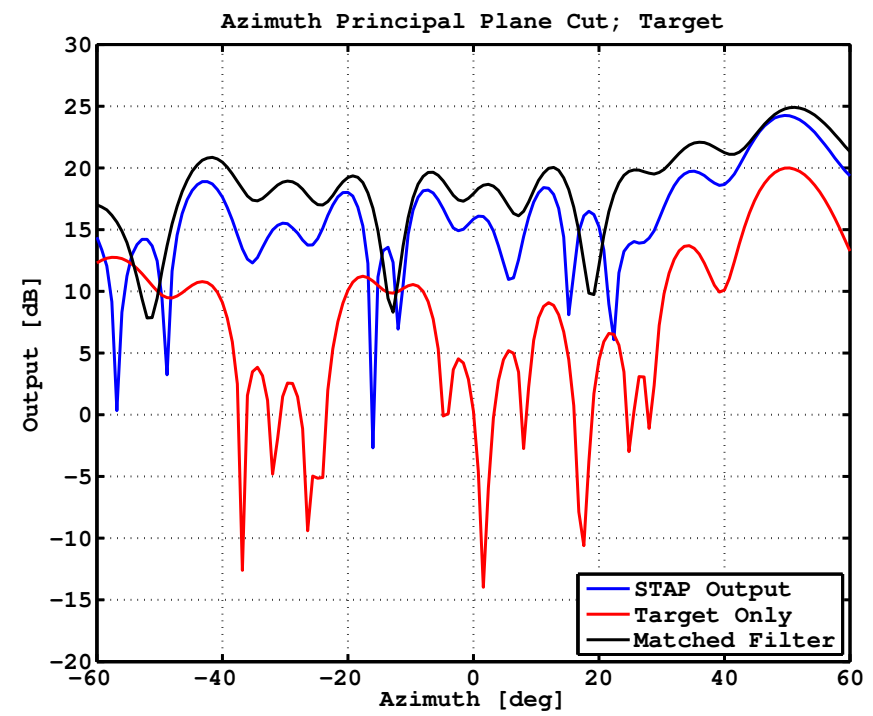

Fig. 7. Beamformer 1; Azimuth Cut, SNR $=20$ dB, 80 Data Snapshots

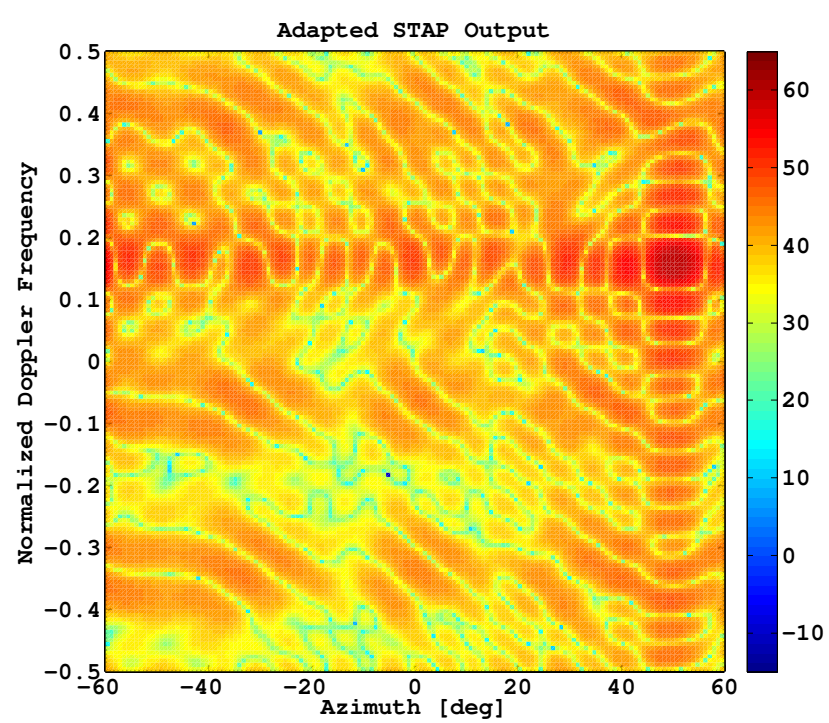

Fig. 9. STAP Output; Beamformer 2, SNR $=60 \mathrm{~dB}, 80$ Data Snapshots

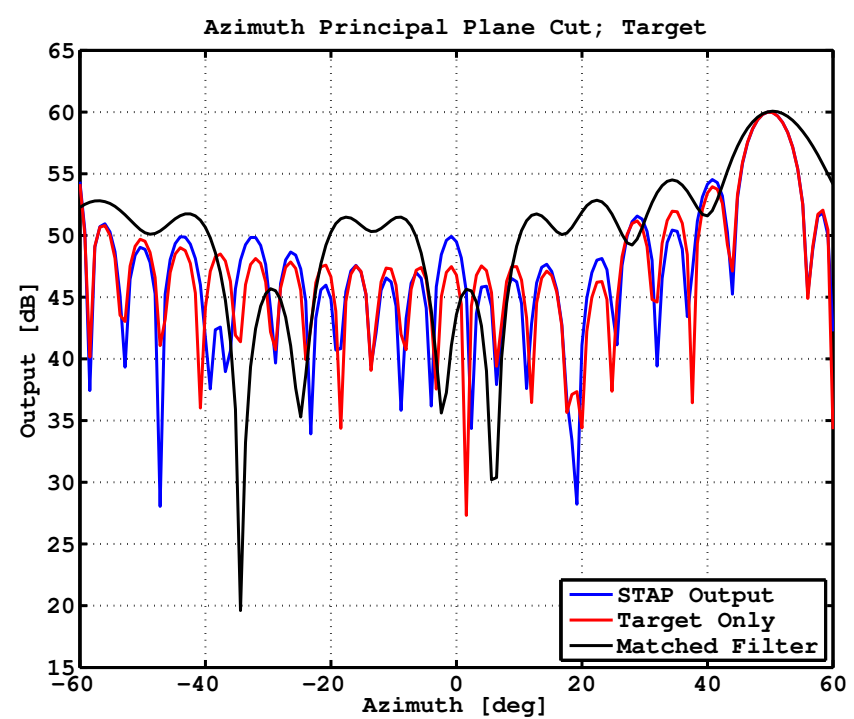

Fig. 11. Beamformer 2; Azimuth Cut, SNR $=60 \mathrm{~dB}, 80$ Data Snapshots

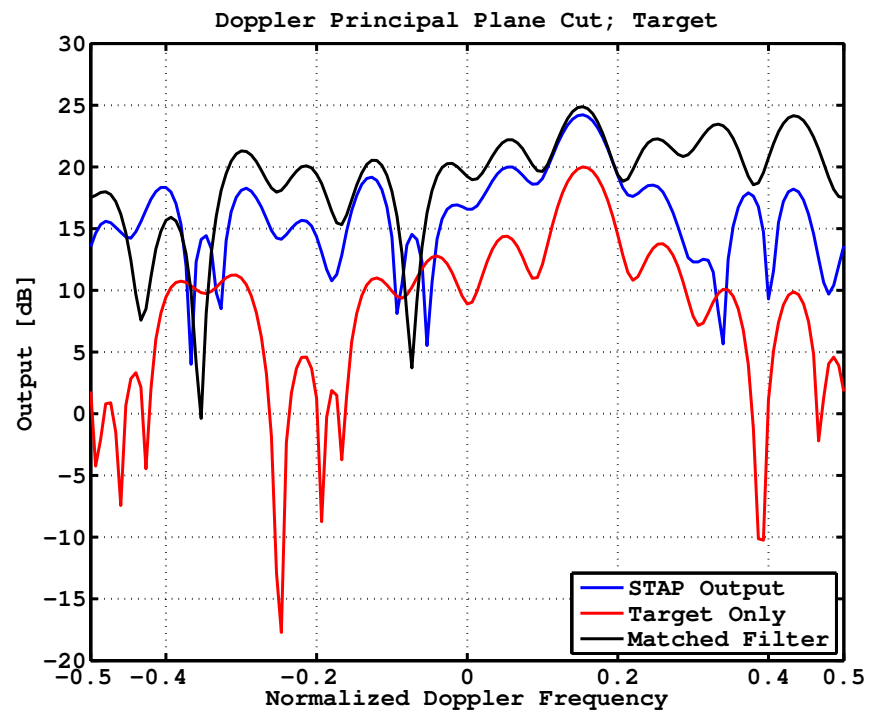

Fig. 8. Beamformer 1; Doppler Cut, SNR $=20$ dB, 80 Data Snapshots

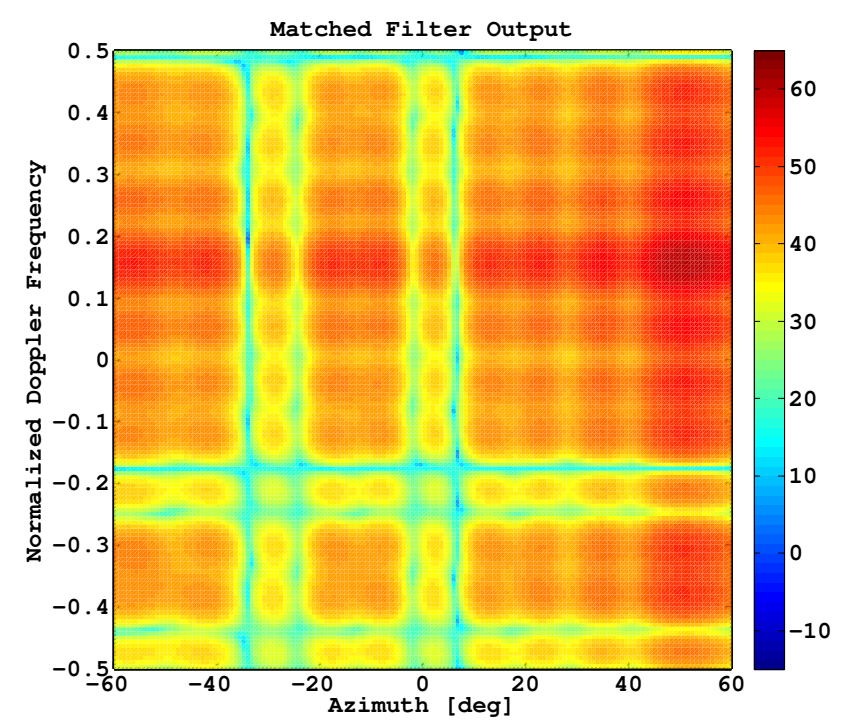

Fig. 10. Matched Filter Output; SNR $=60$ dB, 80 Data Snapshots

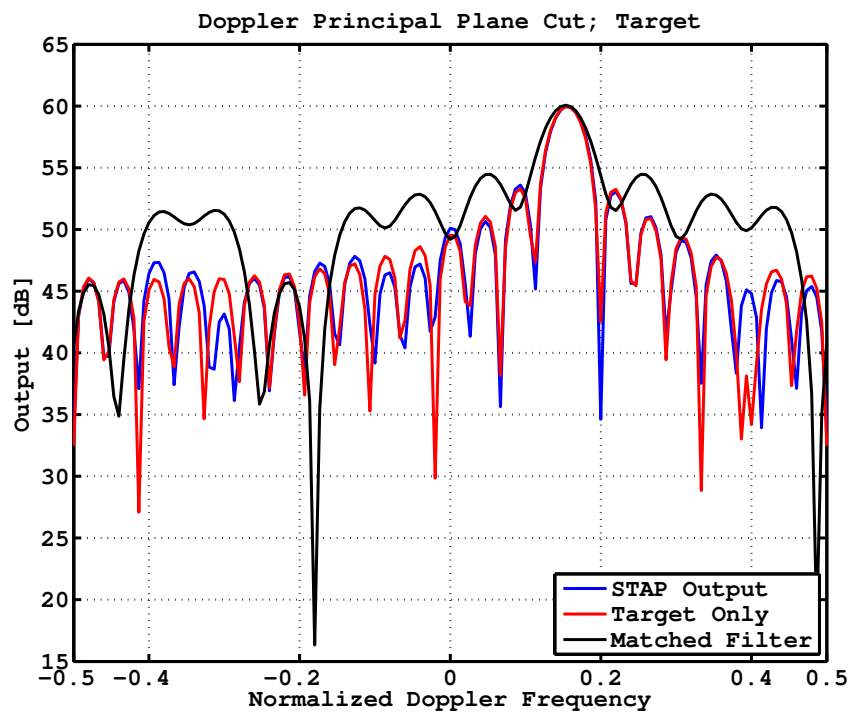

Fig. 12. Beamformer 2; Doppler Cut, SNR $=60 \mathrm{~dB}, 80$ Data Snapshots 